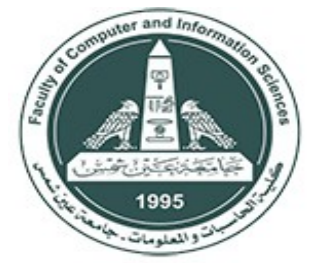

International Joumal of Intelligent Computing and

Information Science

\title{
BUILDING INTELLIGENT SEMANTIC EDUCATIONAL SYSTEM (ISES) BASED ON ONTOLOGY AND SEMANTIC WEB MINING.
}

\author{
Amin, Ahmed E* \\ Department of Computer Science, Mansoura University, \\ Mansoura 35516, Egypt
}

ahmedel_sayed@mans.edu.eg

\begin{abstract}
This research presents a method to build an intelligent semantic education system based on two novel techniques namely ontology and semantic web mining. Ontologies are a powerful mechanism to solve a retrieval problem that is exacerbated rapidly within the modern educational systems, when there are individual differences between instructors and learners in the previous backgrounds and levels of knowledge. In intelligent education systems, four types of ontology are identified that represent knowledge: students, content, education, and structure. There is a promising approach for satisfying the intelligent education systems requirements, which is one of the most prominent semantic Web ideas, based on commonly shared meaning (ontology) and metadata processed automatically. This approach can be supported using semantic querying and conceptual navigation for learning materials.

One of the important results of this paper is the depth of most of the inferences derived from learning ontologies. Relations such as "has Part" and "is Part_Of" and their interplay can be easy dealing by the users. Therefore, this type of thinking can be presented through an automatic mechanism, and semantic information is necessary for reasoning about an automatic occurrence.
\end{abstract}

Keywords: Ontology, Semantic Web, Web Mining, Intelligent Education System.

\section{Introduction:}

The educational organizations are concerned with knowledge management [1] where the learning outcome and their competitiveness can be increased through internal knowledge that is considered as an intellectual asset. So, artificial intelligence techniques are used in the educational institutions to help achieve the aforementioned objectives among these technologies "intelligent systems". In intelligent educational systems, both lecturer and learner contribute to the development of the educational process scenarios. Where, the content is authored which contributes to achieving the goals of learning outcomes by the lecturer whereas, the learner can access to organize the content by selecting educational materials according to his needs and requirements. In order for intelligent educational systems to function satisfactorily, the content must be provided with enrichment information to support indexing and retrieval processes effectively. The structure of most information is currently available in weakly formats such written, audible and visual [2]. From the knowledge management perspective [3], searching for information using traditional keyword-based search engines is one of the limitations of current technology. Information is extracted [4] that required human effort and time to browse the retrieved documents for relevant information and current intelligent agents are unable to perform these tasks satisfactorily. Maintaining of information suffers from stability in terminology and inability to 
remove the outdated information problems. Information uncovering [5] is used to extract implicit knowledge, such as data mining; it is still difficult for poorly distributed document collections. Information viewing depends on the "Views" which usually hide certain information in their databases and make it difficult for the Internet. It is desirable to restricting access to specific information for certain groups of students. In a limited way, the systematic method for describing information resources and thereby improving their access is known as Metadata [6]. Metadata has been used in e-learning communities which were represented in the standard criteria that linked between the content and conditions of use, but it was suffering from a common drawback is the lack of semantics. As a result of the different spatial and temporal sources of information, it is difficult to retrieve and organize learning resources automatically but can fix these problems based on the Semantic Web approach. A Semantic Web [7] is aimed to allow much more advanced knowledge management systems like, including the organization of knowledge in conceptual spaces according to their meaning. Automated tools will support maintenance by checking for inconsistencies and extracting new knowledge. The keywordbased search will be replaced by query answering: requested knowledge will be retrieved, extracted, and presented in a human-friendly way. Query answering over several documents will be supported. Defining who may view certain parts of information (even parts of documents) will be possible. The Semantic Web has two main ideas [8], namely, common shared "ontology" and machine-process able metadata, that allow it develop a promising approach to meet the requirements of intelligent educational systems, where it can support both of the semantic query and the conceptual navigation in the intelligent education systems. There are several factors that are considered to be the strengths of this approach, including the learner's, such the learner's freedom to link the learning materials gathered from different sources with ontologies. Using semantic querying to design courses. The learner can retrieve learning materials in the context of actual problems. Including those related to access to information where the learner can determine the method of access to knowledge according to his interests and needs. The last of these factors is integration where a uniform platform can be provided using Semantic Web to learn integrated activities. The most needed to achieve the Semantic Web technology based on artificial intelligence with the erosion of repeating the mistakes of artificial intelligence, which is represented in the scientific depth, that is the core of the physical and biological problems [9]. In addition, [10] the primary objective of artificial intelligence is to build an intelligent agent that simulates human intelligence, while the goal of the Semantic Web is to help users perform activities on the net. It is clear that the Semantic Web will use the current artificial intelligence technology on a large scale and that progress in this technology will lead to the better Semantic Web. In intelligent education systems, the combination of learning materials becomes difficult because using different sources and terminologies [11]. The apparent disparity in the level and background of knowledge between instructors and learners greatly exacerbates the problem of retrieval. So it was established powerful common understanding mechanism known as "ontology" [12]. In the intelligent education systems must distinguish between the four concepts are Domain Model, Tutorial Model, Student Model and User Interface Model [13]. A content ontology describes the basic concepts of the domain such as computer science and also describes the relationships between these concepts and some basic properties. For example, the study of algorithms is part of mathematics which in turn is part of the Computer Science. The relation "is part of" it should be included by the ontology and the fact that it is transitive. In this way, the agent of automated learning can infer that algorithms of knowledge founded in computer science. The content ontology can also use relations to capture synonyms, abbreviations, and so on. Pedagogy ontology can be used to Pedagogical issues, For example, the scientific material can be presented in the form of a lecture, an example of a tutorial, exercises ... etc. Finally, structure ontology is used to define the logical structure of the learning materials. Typical knowledge of this kind includes hierarchical and navigational relations like previous, next, has Part, is Part Of, requires, and is Based On. Relationships 
can be determined between relations, for example, inverse relations such as has Part and is Part Of. So when developing intelligent education systems we should use the Web ontology language. An ontology and concepts [14-15] are the new tools used in intelligent systems research at present. These terms focus on knowledge sharing and reusability [16]. While the Semantic Web provides tools to help develop educational systems to make them rely on semantics-aware environments. In this context, we consider systematization of the authoring process activities in authoring task ontology as support for better process analysis and more efficient knowledge representation in authoring tools. It provides both methodology and vocabulary [17] for the authoring process description and reasoning. In the end, there is a deep conceptual gap between authoring and authors systems as well as the authoring tools are neither intelligent nor easy to use. Here; the main problem of this paper is crystallized on the possibility of achieving the requirements of the educational environments related to the flexibility of the content and adaptability to the desire of learners and the reusability and the sharing of learning objects and structures as desired by instructors.

The remaining sections of the paper are as follows. The importance of the use of artificial intelligence techniques that represented in semantic web and ontology and its impact of knowledge management in educational institutions was explained in the introduction in section 1. The importance of the semantic web and its architecture is defined, as well as the ontology development in the "Computer Science Department" using the Protégé tool and explained in section 2.Section 3, a brief summary of intelligent educational systems and their components and how they support Semantic Web Services. The method of the proposed system evaluation and the experimental results were discussed in section 4, entitled "Experimental work". Finally, in section 5, a conclusion of the paper followed by the references was presented.

\section{The Related Work:}

Previous studies in the field of intelligent education environments have been concerned with providing an intermediate educational structure characterized by $\sim_{-1}$ intelligence that sets educational goals and provides learning services. Recently, artificial intelligence techniques have been developed that can be used to achieve the previous goals such as:

\subsection{Ontology:}

An ontology term has been defined in several different ways, the definition commonly used is an explicit specification of a set of objects, concepts, and relationships that connect them to each other clearly and accurately in the field of interest [18]. From the definition, we find that this science not only helps the computer to interpret the definitions of concepts in the domain models but also in the creation of relations between them.

An ontology is classified into two categories: the class hierarchy, which includes classes, subclasses, attributes, and values, it can be called Lightweight Ontologies, while the other is the heavy-weight ontology, which includes axioms and constraints [19]. The hierarchical distribution of important concepts in the field and the description of concepts, relationships, and constraints of ontology are the components of the ontology layer as shown in figure 1 [20].

Fig. 1: Ontology and its components [20].

Ontological information will be included in a standard repository for management, storage and archiving [21]. This may be to satisfy legal or operations requirements to maintain version histories. These types of applications require that knowledge engineers interact with subject matter experts to collect knowledge to be captured. 
Ontology in Education (OE) can be viewed from two perspectives depending on Ontology technology kind [22] and Ontology role [23]. The Ontology technology kind perspective can be divided to three main areas, two of which are knowledge representation and information retrieval, while the third one is Semantic Web. As to the application role perspective, Ontologies have been considered for a long time only as a technical artifact acting as a knowledge base component. The field of education is one of the first, where understanding of ontology as a cognitive tool came around. In many respects it was due to the wide spread of the constructivist paradigm of learning and the broad use of such knowledge technologies as concept maps, mind maps and others for learning purposes.

\subsection{Semantic web:}

The World Wide Web (WWW) consists of topics with its own content and similar content is linked to each other through the Universal Resource Locator (URL). The user infers the semantics that describes the surrounding context to communicate the purpose of the link that it represents by the URL. The semantic web term which consists primarily of statements for application consumption to solve the problem of the formal logical structure that not available in the WWW content appeared. The statements are linked together across structures that can form semantics and meaning of the link and may also contain logic that allows for more interpretation and inference of the statements. Semantic Web is the second version of the current web, which represents information not only for use by the computer but also for integration, integration and reuses through applications [25].

Methods of finding and organizing information have evolved significantly using modern web semantic techniques. Semantic Web (SW) relies on semantic web documents, that written using Semantic Web languages like OWL, DAML+OIL. The architecture of the semantic web consists of three layers: the metadata layer, the schema layer, and the logical layer [26]. Metadata layer, known as the popular data model, contains the resource concepts, properties and resource description framework (RDF). Through the schema layer, the concepts are described in a hierarchical format using the Web ontology languages (OWL) and resource description framework schema (RDFS). In the logical layer, a set of modeling primitives is provided for semantic Web that replaces the slow, ineffective, inefficient, and nonintelligent web processes to fast, efficient and inexpensive web processes using a variety of web ontology languages.

Semantic Web (SW) is used in intelligent education systems based on internet to make it more accurate and effective, where offers promising solution to publish information and services on the World Wide Web in a form that is easier for the computers to handle and understand. Where, SW is evolving based on a layered approach and each layer provides a set of functionalities [27]. There are four major layers can be organize the SW technologies namely data and metadata, semantics, enabling technology, and environment [28].

\section{Intelligent Semantic Educational System (ISES) Architecture:}

World Wide Web is considered the greatest potential source for sharing educational information [29]. As shown in figure 2, the proposed system is built on develop approaches like ontology and semantic web that help in solving the problems which arise from the homogeneity of information on structural and semantic level. These problems still occur when syntactic standards are used and let share information be difficult in a meaningful way.

Fig. 2: Intelligent Semantic Educational System Architecture.

Intelligent Semantic Educational System (ISES) consists of hybrid approaches as a starting point that contains three main components: intelligent educational system, Ontology, and Semantic Web analysis. 
The design of ISES is motivated by the potential roles of ontologies in integration of educational information as shown in figure 3. In particular, ontologies are used to represent the intended interpretation of contents different educational information sources. Hybrid approaches are adopted because it provides a good trade-off with respect to development costs and maintainability.

Fig. 3: ISES for ontology based education information sharing

ISES provides the necessary foundation to formulate a query by using a common vocabulary (key words) to determine the required educational objects from various sources of information that available on the Internet and verification of metadata. The shared vocabulary mostly consisted of sequences of hierarchies of concept associated each other by relations. This information is represented by using Resource-Definition Framework (RDF) scheme [30].

In the Semantic Web stage, there is additional layer inserted consists of ontologies and specific structures of information source. The shared vocabulary is used to build the ontologies definitions and the source specification ontologies are encoded by using Web Ontology Language (OWL) which characterized by expressive power that allows to accurately define the intended meaning of the modeling elements and the data values used in the different sources.

\section{Experimental work:}

The proposed intelligent semantic educational system that based on the Semantic Web encompasses efforts to build a new web page architecture that enhances by the educational content with formal semantics. That means, a new vision of learning based on modern techniques used in the field of artificial intelligence and modern educational techniques that take into account the individual differences of students and continuous updating learning sources.

ISES aims to build a new educational structure on the Web enables users to build and manage the content of education meets the needs of learners and develop the performance of teachers and produces an intelligent response to unforeseen situations.

In order to achieve those objectives there are several layers of representational structures are needed such as XML layer, which represents the data structure, the RDF layer, which represents the data mining, the Ontology layer, which represents the formal common agreement about meaning of data, and the Logic layer, which enables intelligent reasoning with meaningful data.

Depending on the previous basic layers above, figure 4 illustrates an architecture of system that consists of a knowledge warehouse that store the metadata repository, the principle inference, and the core modules that are compatible with primary activities in the electronic educational environment such as students modules, content modules and administration modules where the authors can provide the information and both of authors and learners can access to learning materials by querying and browsing.

\section{Fig. 4: ISES Architecture.}

To conduct browse or query for educational material on web pages, the web mining is used. Web Mining is characterized by the ability to apply data mining to obtain on web logs, web contents, and web structures. There are two main components are produced from web mining used mainly in the construction of educational systems namely the web usage mining and the web content mining. The web usage mining is trying to find out what users are looking for while they are using the web, while the web content mining -kind of text mining application- is helping to find the patterns for a particular group of people belonging to a region or depending on their interest. This method could be used for creating metadata for Learning Objects (LO) for building an ontology and semantic structure. 
Ontology is considered the backbone of the system since it has a hierarchy of relevant domain concepts, relations between these concepts, further properties of concepts (attributes with value ranges), and the derivation rules to infer new knowledge.

For example, the ontology of ISES includes classes such as Faculties, Staff, Student, Department and Courses as shown in figure 5. These classes denote concepts and there are subclasses denoted Subconcept such as Stu_Postgraduate is Subclass (Sup-concept) from Student. Each class has a set of instances such as JAVA, AI, and Data Base are instances from Cour_Name Subclass which is Subclass from Courses.

Attributes and relations of concepts are inherited from sub-concepts. Relations refer to other concepts like "has Author" denoting relation between the concept "Cour_Name" and the concept "Professor". Multi-inheritance is supported as a concept may fit into different branches of the taxonomy.

To illustrate ontology, Module students were taken as an example, which consists of four Classes namely Student, Stu_Cour_Info, Stu_Activity, and Stu_Pers_Info. The class “Student" represents any student,

While the class called "Stu_Cour_Info" consists of information about students' performance during the educational process and has a group of subclasses such as Assignment, Cour_Module, Face Meeting, Learning_Obj, and Written_Exam. For details on student activity during the school year, the class "Stu_Activity" is defined. Class "Stu_Pers_Info" represent static and permanent information for students as well as more complex characteristics related to student's interaction with the proposed system ISES. The Protégé tool is used to the ontology development process as shown in figure 6.

Fig. 5: A hierarchy of ISES system classes

Fig. 6: Student Module Ontology (in Protégé)

The Instances member's relationships are modeled as properties of the object. There is a set of object properties like "has $\boldsymbol{A}$ " are used for expressing the linked between the previous characteristics with students, the association of the instances of student characteristics that reflect from classroom like Stu_Cour_Info, Stu_Activity, Stu_Pers_Info, ...etc. with student instances as shown in figure 7. Example for ISES Ontology is shown in figure 8.

Fig. 7: Example of rules extraction from ontology schema and instances.

Fig. 8: ISES Ontology Example.

The concepts sets, concept hierarchy organizing with the best little number from relationships for crosstaxonomical are considered as the output of ontologies. RDF statements are used to represents the set of rules that are enriched from proposed ontology as a knowledge warehouse. The knowledge warehouse itself hosts the ontology, the metadata, as well as the data proper. The knowledge warehouse is enable inference mechanisms to answers queries and it performs derivations of new knowledge by an intelligent combination of facts in the knowledge warehouse with the ontology. Figure 9 is shown examples for the ISES rules expressed in semantic web rules language.

Fig. 9: Snapshot of ISES Rules.

\subsection{ISES Evaluation:}


There are many measures and methods used to examine a set of criteria that used in ontology evaluation. Both of measures and methods basically differ not only on how many of these criteria are targeted but also their main motivation behind evaluating the taxonomy. Four approaches are used to classify these measures and methods namely [31] gold standard approach, the approach based on a datadriven, the approach based on task and the approach based on criteria.

The approach based on the gold standard [32], which is also known as ontology alignment or ontology mapping is the most straight-forward approach. An idealized outcome of the learning algorithm is represented by using a comparison between the proposed ontology with a previously created reference ontology. Both layers, lexical term, and concept hierarchy of an ISAS ontology are evaluated by using this approach. The idea of precision and recall to the gold standard based evaluation of ontologies is extended from the measures. The information retrieval techniques are Precision (Prec), Recall (Rec), and F-measure (F). The reference retrieval $\left(\operatorname{Ret}_{R}\right)$ and the computed retrieval $\left(\operatorname{Ret}_{C}\right)$ returned by a system are compared by information retrieval techniques. Precision and recall are defined as follows:

$$
i\left(\operatorname{Ret}_{R}, \operatorname{Ret}_{C}\right)=\frac{\left|\operatorname{Ret}_{C} \cap \operatorname{Ret}_{R}\right|}{\left|\operatorname{Ret}_{C}\right|} \quad, \quad \operatorname{Rec}\left(\operatorname{Ret}_{R}, \operatorname{Ret}_{C}\right)=\frac{\left|\operatorname{Ret}_{C} \cap \operatorname{Ret}_{R}\right|}{\left|\operatorname{Ret}_{R}\right|}
$$

Since, precision and recall are opposite each other can infer the following equation:

$$
i\left(\operatorname{Ret}_{R}, \operatorname{Ret}_{C}\right)=\frac{\left|\operatorname{Ret}_{C} \cap \operatorname{Ret}_{R}\right|}{\left|\operatorname{Ret}_{C}\right|}=\operatorname{ReC}\left(\operatorname{Ret}_{C}, \operatorname{Ret}_{R}\right)
$$

The F-measure is the harmonic mean of $i$ and Rec , which used for giving overview summary and balance the $i$ and Rec values. It's calculated by:

$$
F\left(\operatorname{Ret}_{R}, \operatorname{Ret}_{C}\right)=\frac{2 . \prec\left(\operatorname{Ret}_{R}, \operatorname{Ret}_{C}\right) \cdot \operatorname{Rec}\left(\operatorname{Ret}_{R}, \operatorname{Ret}_{C}\right)}{i\left(\operatorname{Ret}_{R}, \operatorname{Ret}_{C}\right)+\operatorname{Rec}_{\left(\operatorname{Ret}_{R}, \operatorname{Ret}_{C}\right)}}
$$

Evaluation of ontology can be divided into lexical term and concept hierarchy. Precision and recall are used to evaluate the lexical term are defined as the following:

$$
\begin{aligned}
& \text { Lixical }_{(}\left(\text {Ontology }_{C}, \text { Ontology }_{R}\right)=\frac{\left|C_{C} \cap C_{R}\right|}{\left|C_{C}\right|} \\
& \text { Lixical }_{\text {Rec }}\left(\text { Ontology }_{C}, \text { Ontology }_{R} \mid=\frac{\left|C_{C} \cap C_{R}\right|}{\left|C_{R}\right|}\right.
\end{aligned}
$$

Where; Ontology $y_{c}$ and Ontology ${ }_{R}$ are computed core ontology and reference ontology respectively. $C_{C}$ and $C_{R}$ are computed retrieval concept and reference retrieval concept respectively. Whereas evaluation of concept hierarchy can be defined as a family of measures that used for systematically constructing a measure. There are two kinds of measures namely local and global used to a comparison between two concept hierarchies with each other. The local measure is used to compares the positions of two concepts whereas the global measure is using for whole concept hierarchies comparing.

The precision of the local taxonomic is computed by the two concepts similarity based on the extracted characteristic from the concept hierarchy. A characteristic extract $\left(C_{E}\right)$, the local taxonomic precision

$\left(\dot{i}_{\text {Pres }}\right)$ of two concepts $C_{1} \in$ Ontology $_{C}$ and $C_{2} \in$ Ontology $_{R}$ is defined as:

$\dot{i}_{i}\left(C_{1}, C_{2}\right.$, Ontology $_{C}$, Ontology $\left._{R}\right)=\frac{\mid C_{E}\left(C_{1}, \text { Ontology }_{C}\right) \cap C_{E}\left(C_{2}, \text { Ontology }_{R}\right) \mid}{\mid C_{E}\left(C_{1}, \text { Ontology }_{C}\right) \mid}$ 
The super and sub level that known as semantic couple $\left(\operatorname{Sem}_{C o}\right)$ is measured by the taxonomic overlap measure [33] to characterize a concept. Given the concept $c \in C$ and the ontology $O$, the semantic couple $\mathrm{Sem}_{\mathrm{Co}}$ is defined as follows:

$$
\operatorname{Sem}_{C o}(c, O)=\left\{c_{i} \mid c_{i} \in C \wedge\left(c_{i} \leq c \vee c \leq c_{i}\right)\right\}
$$

When using the common semantic pair $\left(\right.$ ComSem $\left._{C o}\right)$ as a characteristic extract, the influence of lexical precision and recall on the taxonomic measures are avoided. All concepts which are not also available in the other ontology's concepts set are excluded by the common semantic couple:

ComSem $_{C o}\left(c, O_{1}, O_{2}\right)=\left\{c_{i} \mid c_{i} \in C_{1} \cap C_{2} \wedge\left(c_{i}<1 c \vee c<1 c_{i}\right)\right\}$

A global taxonomic precision $\left(\dot{b}_{\text {Pres }}\right)$ measure can be used now to define a framework for constructing.

$$
\dot{i}_{\text {Pres }}\left(\text { Ontology }_{C}, \text { Ontology }_{R}\right)=\frac{1}{\left|C_{c}\right|} \sum_{\begin{array}{c}
\text { Concept Set } \\
c \in C_{c}
\end{array}}\left\{\begin{array}{c}
i_{\text {Pres }}\left(c, c, \text { Ontology }_{C}, \text { Ontology }_{R}\right) \text { if } c \in C_{R} \\
\max _{\dot{c} \notin C_{R}} \dot{i}_{\text {Pres }}\left(c, \dot{c}, \text { Ontology }_{C}, \text { Ontology }_{R}\right) \text { if } c \notin C_{R}
\end{array}\right\}
$$

From the previous equation is precise taxonomic concepts proposed for all the expense and if the accuracy taxonomic value equal to zero, this means that the current concept is not contained in the reference concepts as:

$$
\begin{aligned}
& \dot{i}_{i}\left(\text { Ontology }_{C}, \text { Ontology }_{R}\right)=\frac{1}{\left|C_{c}\right|} \sum_{c \in C_{c}}\left\{\begin{array}{c}
i_{\text {Pres }}\left(c, c, \text { Ontology }_{C}, \text { Ontology }_{R}\right) \text { if } c \in C_{R} \\
0 \text { if } c \notin C_{R}
\end{array}\right. \\
& \dot{i}_{\text {Recall }\left(\text { Ontology }_{C}, \text { Ontology }_{R}\right)=\dot{i}_{i}\left(\text { Ontology }_{R}, \text { Ontology }_{C}\right)}
\end{aligned}
$$

A comprehensive analysis of the learned ontologies is required to conduct the evaluation process, so the

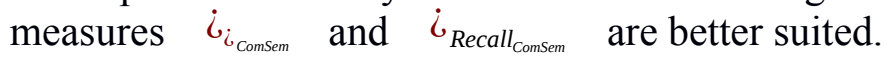

$$
\begin{aligned}
& \dot{i}_{\text {Pres }_{\text {Consem }}}\left(\text { Ontology }_{C}, \text { Ontology }_{R}\right)=\frac{1}{\left|C_{c} \cap C_{R}\right|} \sum_{c \in C_{c} \cap C_{R}} i_{i_{\text {consem }}}\left(c, c, \text { Ontology }_{C}, \text { Ontology }_{R}\right) \\
& \dot{i}_{\text {Recall }_{\text {consem }}}\left(\text { Ontology }_{C}, \text { Ontology }_{R}\right)=\dot{i}_{\dot{i}_{\text {Consem }}\left(\text { Ontology }_{R}, \text { Ontology }_{C}\right)}
\end{aligned}
$$

The harmonic mean of the global taxonomic precision and recall is known as the taxonomic F-measure (TF).

$$
T F\left(\text { Ontology }_{C}, \text { Ontology }_{R}\right)=\frac{2 . \dot{i}_{i}\left(\text { Ontology }_{C}, \text { Ontology }_{R}\right) \cdot \dot{i}_{\text {Recall }}\left(\text { Ontology }_{C}, \text { Ontology }_{R}\right)}{\dot{i}_{i}\left(\text { Ontology }_{C}, \text { Ontology }_{R}\right)+\dot{i}_{\text {Recall }}\left(\text { Ontology }_{C}, \text { Ontology }_{R}\right)}
$$

The local overlap is used instead of the local taxonomic precision in the global taxonomic overlap (TO) which has the same building blocks like TP, to:

$$
i=\frac{T F}{2-T F}
$$

\subsection{Experimental and Discussion:}

This paper uses, HERO [34] ontology as reference ontology, which stands for "Higher Education Reference Ontology" as shown in figure 10. HERO ontology is projected to be a reusable and generalizable resource of academic knowledge which can be filtered to meet the needs of any knowledge-based application that requires structural information.

Fig. 10. Reference Ontology ( Ontology ${ }_{R}$, left) and Proposed Computed Ontology ( Ontology ${ }_{C}$, right). 
Tables 1 and 2 are shown the influence of inserting and replacing concepts in a hierarchy. The tables contain the sets lexical (SC) and Common Semantic couple (ComSem) for the ontologies Ontology $_{R}$ and Ontology . $_{\text {. }}$

Table 1. Semantic copies for the ontologies in Fig. 10

Table 2. Common semantic copies for the ontologies in Fig. 10

From tables 1 and 2, showed that the common semantic couple is not affected by the replacing and inserting of concepts without a real change in the hierarchy, while they are strongly influenced by changes in the lexical term layer of an ontology.

When comparing with the Ontology $_{R}$ (HERO) there are many concepts missing in Ontology ${ }_{C}$ (ISES) not only the natural language identifier of some concepts changes but also the hierarchy of remaining concepts.

In Table 3 , It is expected that the lexical precision and recall of the measures $\dot{i}_{\text {Pres }_{\text {Consem }}}$ and $\dot{i}_{\text {Recall }}$ consem are independent depending on the properties of the common semantic couple of the taxonomic measures.

Table. 3: Evaluation of the ontologies in Fig. 10 with $\operatorname{Sem}_{\mathrm{Co}}$ and $\operatorname{ComSem}_{\mathrm{Co}}$.

It is surprising that, the lexical precision and recall influence Lixical $_{i}$ and Lixical $_{\text {Rec }}$. Table 3 shows the significant decrease between the taxonomic measures and the lexical measures, which leads to the visibility of errors in the lexical term layer of learned ontology when comparing the values both of the taxonomic and lexical measures.

\section{Conclusion:}

This paper presents a proposal for integration between two novel techniques Ontology and Semantic Web. The effect of both techniques on intelligent semantic education systems is discussed, which have already become the basic requirements in education. The integration of semantic rules is one of the main advantages of the proposed system. All of the students, content, pedagogy, and structure that are already incorporated in the ontology are classified by the rules combined with inference mechanisms. To evaluate the ISES ontologies, a multidimensional evaluation was used named a gold standard based evaluation. Based on the concept position in the hierarchy, the weighting of the errors was confirmed in comparison to the existing measures, where the scale interval was used in a more evenly.

\section{Reference:}

1. Alavi, M., \& Leidner, D. E. (2001). Knowledge management and knowledge management systems: Conceptual foundations and research issues. MIS quarterly, 107-136.

2. Garner, W. R. (2014). The processing of information and structure. Psychology Press.

3. Kim, T. H., Lee, J. N., Chun, J. U., \& Benbasat, I. (2014). Understanding the effect of knowledge management strategies on knowledge management performance: A contingency perspective. Information \& management, 51(4), 398-416.

4. Kara, S., Alan, Ö., Sabuncu, O., Akpınar, S., Cicekli, N. K., \& Alpaslan, F. N. (2012). An ontology-based retrieval system using semantic indexing. Information Systems, 37(4), 294-305.

5. Starik, M., \& Kanashiro, P. (2013). Toward a theory of sustainability management: Uncovering and integrating the nearly obvious. Organization \& Environment, 26(1), 7-30. 
6. Scheer, A. W. (2012). Architecture of integrated information systems: foundations of enterprise modelling. Springer Science \& Business Media.

7. Navigli, R., \& Velardi, P. (2004). Learning domain ontologies from document warehouses and dedicated web sites. Computational Linguistics, 30(2), 151-179.

8. Zeng, K., Yang, J., Wang, H., Shao, B., \& Wang, Z. (2013, February). A distributed graph engine for web scale RDF data. In Proceedings of the VLDB Endowment (Vol. 6, No. 4, pp. 265-276). VLDB Endowment.

9. Isotani, S., Mizoguchi, R., Isotani, S., Capeli, O. M., Isotani, N., De Albuquerque, A. R. ... \& Jaques, P. (2013). A Semantic Web-based authoring tool to facilitate the planning of collaborative learning scenarios compliant with learning theories. Computers \& Education, 63, 267-284.

10. Frensch, P. A., \& Funke, J. (2014). Complex problem solving: The European perspective. Psychology Press.

11. Polson, M. C., \& Richardson, J. J. (2013). Foundations of intelligent tutoring systems. Psychology Press.

12. Wimalasuriya, D. C., \& Dou, D. (2010). Ontology-based information extraction: An introduction and a survey of current approaches.

13. Romero, C., \& Ventura, S. (2010). Educational data mining: a review of the state of the art. IEEE Transactions on Systems, Man, and Cybernetics, Part C (Applications and Reviews), 40(6), 601-618.

14. Barnaghi, Payam, et al. "Semantics for the Internet of Things: early progress and back to the future." International Journal on Semantic Web and Information Systems (IJSWIS) 8.1 (2012): $1-21$.

15. Mizoguchi, R., \& Bourdeau, J. (2000). Using ontological engineering to overcome common AIED problems. Journal of Artificial Intelligence and Education, 11, 107-121.

16. Chen, W., Hayashi, Y., Jin, L., Ikeda, M., \& Mizoguchi, R. (1998). An ontology-based intelligent authoring tool. Proceedings of ICCE'98, 41-49.

17. Mizoguchi, R., Sinitsa, K., \& Ikeda, M. (1996, June). Task ontology design for intelligent educational/training systems. Position Paper for ITS'96 Workshop on Architectures and Methods for Designing Cost-Effective and Reusable ITSs, Montreal.

18. Wei, W., \& Gulla, J. A. (2010, July). Sentiment learning on product reviews via sentiment ontology tree. In Proceedings of the 48th Annual Meeting of the Association for Computational Linguistics (pp. 404-413). Association for Computational Linguistics.

19. Jain, V., \& Singh, M. (2013). Ontology development and query retrieval using protégé tool. International Journal of Intelligent Systems and Applications (IJISA), 5(9), 67.20.

20. http://semanticweb.org/wiki/Semantic_Web.

21. Sánchez, D. (2010). A methodology to learn ontological attributes from the Web. Data \& Knowledge Engineering, 69(6), 573-597.

22. Al-Safadi, L. A., \& Al-Abdullatif, N. A. (2010). Educational advertising ontology: a domaindependent ontology for semantic advertising networks. Journal of Computer Science, 6(10), 1070.

23. Giri, K. (2011). Role of ontology in semantic web. DESIDOC Journal of Library \& Information Technology, 31(2).

24. Tsytsarau, M., \& Palpanas, T. (2012). Survey on mining subjective data on the web. Data Mining and Knowledge Discovery, 24(3), 478-514.

25. D. Lavbic, I. Lajovic, and M. Krisper, Facilitating information system development with panoramic view on data, Computer Science and Information Systems 7 (2010), 737-767. 
26. Bucos, M., Dragulescu, B., \& Veltan, M. (2010, November). Designing a semantic web ontology for E-learning in higher education. In 2010 9th International Symposium on Electronics and Telecommunications (pp. 415-418). IEEE.

27. Mishra, R. B., \& Kumar, S. (2011). Semantic web reasoners and languages. Artificial Intelligence Review, 35(4), 339-368.

28. Larman, C. (2012). Applying UML and patterns: an introduction to object oriented analysis and design and interative development. Pearson Education India.

29. Aghaei, S., Nematbakhsh, M. A., \& Farsani, H. K. (2012). Evolution of the World Wide Web: From WEB 1.0 TO WEB 4.0. International Journal of Web \& Semantic Technology, 3(1), 1.

30. Bernstein, D., \& Vij, D. (2010, July). Intercloud directory and exchange protocol detail using XMPP and RDF. In 2010 6th World Congress on Services (pp. 431-438). IEEE.

31. Tartir, S., Arpinar, I. B., \& Sheth, A. P. (2010). Ontological evaluation and validation. In Theory and applications of ontology: Computer applications (pp. 115-130). Springer, Dordrecht.

32. Timmermans, S., \& Berg, M. (2010). The gold standard: The challenge of evidence-based medicine and standardization in health care. Temple University Press.

33. Maedche, A., \& Staab, S. (2002, October). Measuring similarity between ontologies. In International Conference on Knowledge Engineering and Knowledge Management (pp. 251263). Springer, Berlin, Heidelberg.

34. Zemmouchi-Ghomari, L., \& Ghomari, A. R. (2013). Process of building reference ontology for higher education. In Proceedings of the World Congress on Engineering (Vol. 3, pp. 1595-1600). 